Check for updates

Cite this: Phys. Chem. Chem. Phys., 2020, 22, 6011

DOI: $10.1039 / \mathrm{d} 0 \mathrm{cp} 90055 \mathrm{a}$

rsc.li/pccp

\section{Correction: Predictable spectroscopic properties of type-II ZnTe/CdSe nanocrystals and electron/ hole quenching}

\author{
Tongqing Long, ${ }^{\mathrm{a}}$ Jun $\mathrm{CaO}^{\mathrm{a}}$ and Zhong-Jie Jiang (D)*ab
}

Correction for 'Predictable spectroscopic properties of type-II ZnTe/CdSe nanocrystals and electron/ hole quenching' by Tongqing Long et al., Phys. Chem. Chem. Phys., 2019, 21, 5824-5833.

The authors apologize that it was not made clear to readers that the same sample originally reported in the corresponding author's previous work reported in J. Phys. Chem. $C$ (cited as ref. 23 in the original article) was used for comparison in this Physical Chemistry Chemical Physics article. ${ }^{1}$

The ZnTe/CdSe nanocrystals with $2.65 \mathrm{~nm}$ ZnTe core reported in this article are the same as the ZnTe/CdSe nanocrystals with $2.6 \mathrm{~nm}$ ZnTe core that were originally reported in ref. 1. However, in this article, the size of the ZnTe core and the CdSe shell thicknesses have been re-measured to provide more accuracy.

There is a lot of new work reported in this paper, although we have used the same sample that was reported in the previous work for comparison. That is because we wanted to give a clearer comparison to demonstrate that our model can be applied to predict the spectroscopic properties of particles with different core sizes. This gives better support to our finding that the spectroscopic properties can be precisely predicted. In this article, we mainly focus on demonstrating the precision of the predicted conduction and valence band edges, so the photoluminescence quenching is systematically investigated. The significant innovation and insight into physical chemistry in this work are different from the previous paper.

In this article, new data have been added to each figure, and the old data, which have been re-analyzed and re-plotted, are only used as a comparison. The authors apologise that this was not made clear in the figure captions.

Fig. 1d was originally published in ref. 1 (Fig. S1e).

For Fig. 1a and 2a, the spectrum data are from the same sample as ref. 1, although we re-measured the sizes of the particles by selecting more particles for statistical analysis. Although it is the same sample, the spectrum data are re-analyzed and re-plotted.

For Fig. 3a-c, new experimental data were added to the current work, and the data of the sample that was reported in ref. 1 were obtained by recalculation and used only as a comparison.

The Royal Society of Chemistry apologises for these errors and any consequent inconvenience to authors and readers.

\title{
References
}

1 Z.-J. Jiang and D. F. Kelley, J. Phys. Chem. C, 2012, 116, 12958-12968.

\footnotetext{
${ }^{a}$ Guangzhou Key Laboratory for Surface Chemistry of Energy Materials, New Energy Research Institute, College of Environment and Energy, South China University of Technology, Guangzhou 510006, China. E-mail: eszjiang@scut.edu.cn

${ }^{b}$ Guangdong Engineering and Technology Research Center for Surface Chemistry of Energy Materials, New Energy Research Institute, College of Environment and Energy, South China University of Technology, Guangzhou 510006, China
} 